\title{
Methodology Design of Computational Experiments Founded on Design-based Science Case Study: Motion Models
}

\author{
http://dx.doi.org/10.3991/ijoe.v12i07.5850 \\ John Petearson Anzola, Helien Parra Riveros and Andres Camilo Jimenez \\ Fundación Universitaria Los Libertadores, Bogotá D.C, Colombia
}

\begin{abstract}
In this article, using design methodology and design-based science, random waypoint motion models and Manhattan against a model of semicircular motion are compared. The analysis involved the total distance traveled, the average total energy of robot agents, and convergence time at $80 \%$ of exploration of the navigation area. Robot agents involved in the experiment show no cognitive and cooperative parameter. Therefore, the results obtained in this article will allow extracting temporal and energy characteristics that allows exploring as much area in a shortest possible time in order to get heuristics for future work in SLAM (Simultaneous Localization and Mapping) tasks.
\end{abstract}

Index Terms-Design-based science, motion models, NetLogo, agent-based system.

\section{INTRODUCTION}

A technological aspect present in terrain recognition systems and space deals with localization tasks and simultaneous mapping. The objective is to discover whether it is possible for a mobile robotic agent to navigate through an unfamiliar environment and build a map incrementally, so that at the same time it can determine its position within the map.

One of the main applications of research and development corresponds to cooperative networks through a communication protocol, shared processing information, location, and mapping.

In this article the use of the two most widespread motion stochastic models are analyzed. These are random waypoint motion model and Manhattan; along with a semicircular motion model, where the movement is performed in closed trajectories.

The motion model plays an important role since these models can define energy efficiency in SLAM tasks in decentralized and distributed environments, where disclosure is given in an ad hoc form. The energy in this type of scenario is directly proportional to the total distance traveled. Therefore, it is necessary to evaluate the movement characteristics of the robotic agents before starting SLAM tasks and adding intelligence and perception to robotic units.

To compare these motion models, based on the methodology of design-based science, a computational experiment series were designed. This methodology allows one to create and guide the design of a computational experiment. The aim of the methodology is to compare the random waypoint motion models (Manhattan and semicircular), evaluating the performance measures, accumulated distance, power consumed of all nodes (robots), and average shocks expected in each motion model.

\section{Methodology (Design-BASED Science)}

Within the methodology of design-based science it is suitable to meet at least two conditions:

i) The methodology must be able to search through a range of solutions. Out of those one or more are not biased by experience or intuition of the designer, creating "new alternatives" that were unknown.

ii) Design alternatives must be repeatable and reproducible in order to adequately control the variables that influence the design.

In a design of computational experiments, the set of operations and relationships between data must converge to the algorithm design or procedures. The design must be developable and programmable in computational tools, while satisfying the above conditions.

In the methodology of design-based science, designs of computational experiments are developed in a way that allows to increase quality and reduce the design time depending on the experiment design complexity. The design of hierarchical procedures is usually an appropriate choice in the framework of the experimental design, as it is constructed deductively, starting from the general components to design specific components [1], [2]. Furthermore, the experiment results are described inductively based on the specificity of the results.

At the different levels each component is generated through a complete design specification. The procedures are iterative where convergence properties are optimality determined. A general model is described in Figure 1.

As input parameters, the model has a research module and requirements. For a given experiment, the research module is introduced with the problem to evaluate its characteristics and possible quality metrics. As a requirement, the module identifies restrictions applicable to the experiment and its possible limitations; whether physical, spatial, etc.

The design module, contemplates the algorithms and processes to be implemented, whether in software and hardware. The programing language is defined to create the functions of the experiment and is iteratively tested each of the units and sub-units. It defines the design to ensure that the implementation is in accordance with requirements. The output of the methodology allows observing the computational assessment of the experiment using visual analytics tools. 


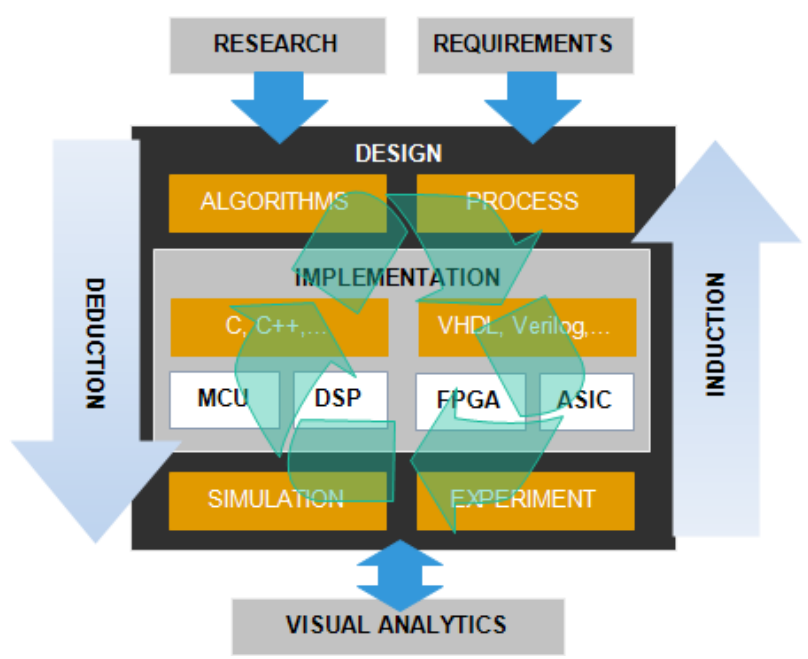

Figure 1. Methodology for design of computational experiments founded on design-based science

\section{A. Research}

For the design of computational experiments of random mobility we have selected three movements:

i) Random waypoint motion model [3]

ii) Manhattan motion model [4]

iii) Semicircular motion model

The model of semicircular mobility is the proposed model as a new alternative to traditional models.

\section{B. Requirements}

As requirements we have the creation of a stage that has an area of $50 \times 50$ units and each unit has a measure of $10 \mathrm{~cm}$. The perimeter of the stage has a width of $10 \mathrm{~cm}$ (shown in black) and the robots cannot pass this limit. This width will allow the robot go backward if the inertia of the robot moves it off the stage. The stage described above is shown in Figure 2.

In Figure 2, the shaded area in red correspond to area already traveled and explored by the robot. Where the white area are not yet explored. The convergence parameter of the experiment was taken as a rule to stop the simulation process, once the robot has made the route or explored $80 \%$ of the total area.

\section{Design}

Once the motion models and restrictions were defined, we proceed to define the processes and algorithms required for the experiment design. In this section of the experiment (Figure 1), the methodology defines the programing language to be used, in this case it is a NetLogo. The NetLogo is a programming environment of agent models that allows simulating physical, natural, and social phenomena.

NetLogo as an implementation tool for the modeling that allows passing instructions to hundreds or thousands of "agents" simultaneously or schedule specific behaviors to a group of certain agents [5]. For the experiment raised, each agent represents a robot, enabling to explore the behavior relations of one or dozens of robots.

\section{Visual Analytics}

This methodology section refers to the output data of the experiment, where performance metrics of the experiment are expressed as well as evaluated the motion mod-

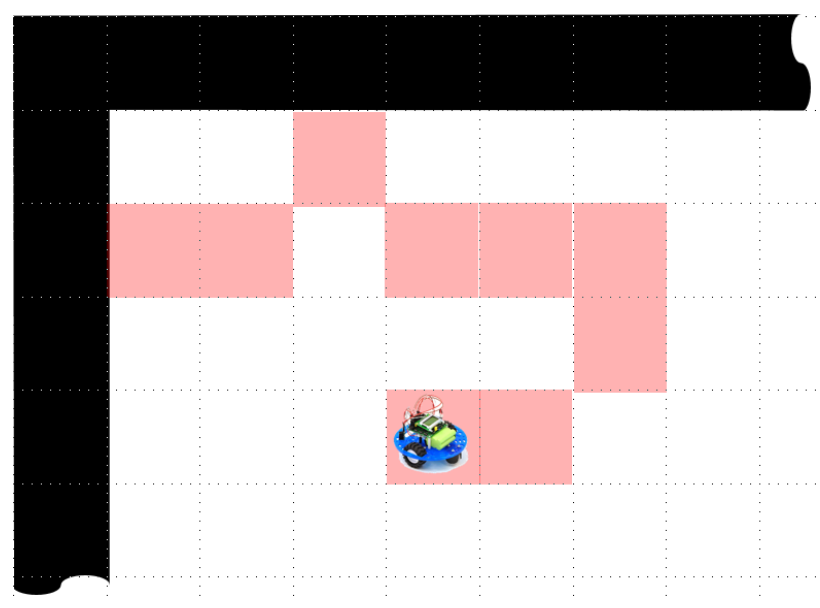

Figure 2. Simulation Stage (area scanned by a robot)

els. For the experiment, the following performance metrics were chosen:

i) Accumulated distance by the total number of agents

ii) Total energy consumed by all agents

iii) Number of shocks for each motion model

iv) Exploration convergence time at $80 \%$

The methodology for designing computational experiments were described in this section by following a deductive approach. It demonstrates that the random waypoint stochastic model and Manhattan model are the most representative in simulation schemes. At this point, postulated as hypothesis of a semicircular motion model, which in contrast to the random waypoint motion models and Manhattan model. It is estimated that the semicircular motion model would present a better performance than the two other proposed models.

Consequently, the results obtained and contrasted serve as evidence to establish whether the hypothesis with the new model has better performance than the other two models. Using a process of deduction, the results of visual analytic can be explained whether the hypothesis can be considered wrong (wholly or partly).

\section{MOTION MODELS}

\section{A. Random waypoint motion model}

The applications of the Random Waypoint motion model have been used to evaluate the performance of routing protocols in dynamic environments extended in MANETs networks. In this model, the position of each robot agent is selected randomly and then moved to the selected position linearly with constant random speed [6].

This move was designed to have a pause time before starting the next move. The pause time is determined by the model initialization and its speed is uniformly distributed between zero and maximum. The movement of each node has a linear direction except when the movement is outside the working area limit; given the case it is reflected and its direction has changed.

The random waypoint motion model is modified so that it can use in the stage as presented in Figure 2. The robot agent will move about $10 \mathrm{~cm} / \mathrm{s}$, each of these movements will be equivalent to one unit in the NetLogo environment simulation. 


\section{B. Manhattan motion model}

In the Manhattan motion model, known as maps of city streets, has been used to generate mobility of nodes, where top-view plan of a city is composed of vertical streets, horizontal streets, and their intersection [7]. In this model, a node is placed randomly anywhere on the plan. By the time the node starts to move horizontally or vertically and reaches the interception of a street. Once the node is at the street intersection, it must make a random decision between turning right, left or go straight.

If a node wants to move in the same direction, then the node has a 0.5 of probability to continue in the same direction or 0.25 probability of turning left or right. The speed depends on the direction of the previous movement.

In this case, the stage is free of obstacles. Therefore, this model was modified leaving the chance so the robot agent can go backward. In that case the weight of the four possibilities; forward, backward, left and right have a probability of 0.25 , avoiding the loop modification in the corners.

\section{Semicircular motion model}

This model implemented in this article is a variation of the random waypoint motion model, where instead of taking a random angular position of 360 degrees, it takes an angular position of 45 degrees. This variation makes the semi-circular model of incremental trajectories free of loops in critical areas, such as the corners of the stage.

In Figure 3, the path of a node in the proposed stages is observed graphically.
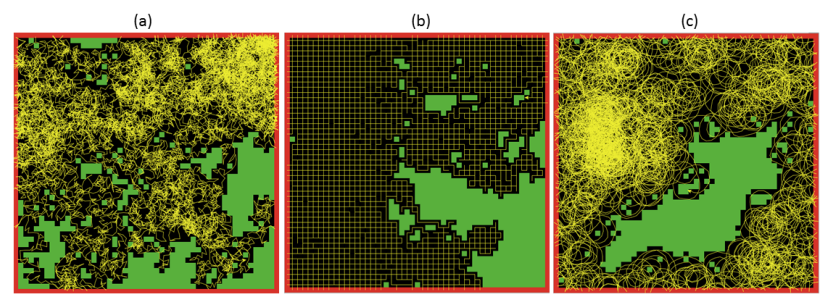

Figure 3. Movements: a) Random waypoint, b) Manhattan and c) semicircular

\section{DESCRIPTION OF THE EXPERIMENT}

In Figure 3, the trajectories of motion models to be evaluated are observed. The perimeter of the stage in NetLogo is red, the unexplored area is green and the scanned area is black. In NetLogo each of the "turtle" agents represent the robots. The agents are programmed for each motion models and is evaluated the distance traveled, estimated energy consumed by all agents and the number of shocks that presents each model.

The average energy model was estimated with the maximum operating parameters of the robot "Popolu 3pi", which on average consumes $600 \mathrm{mAH}$, where the programmed speed of agents corresponds to $10 \mathrm{~cm} / \mathrm{s}$.

The total area of agents' movement corresponds to $50 \times 50$ units of $10 \mathrm{~cm}$, for a total of $5 \mathrm{~m}^{2}$.

Part of this article's purpose is to evaluate the performance of motion models, from 1 agent to 50 agents, with a stepped projection of $60,70,80,90$ and 100 agents.

\section{Results OBTAined}

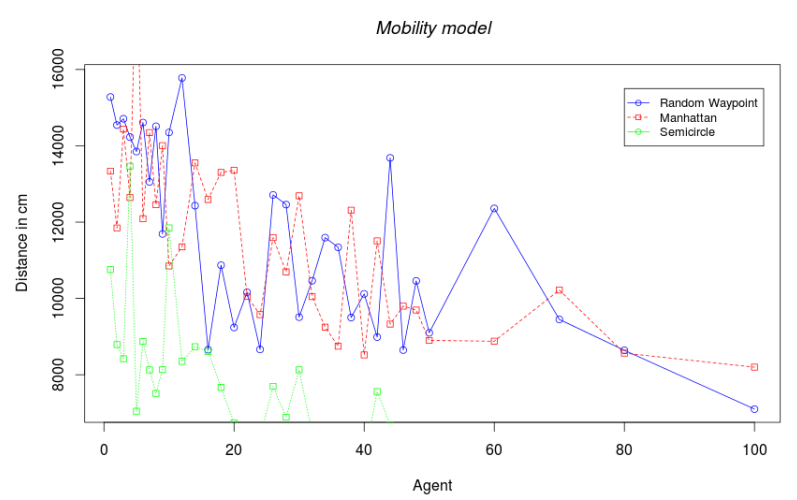

Figure 4. Distance in $\mathrm{cm}$ traveled by the number of agents, with $80 \%$ convergence of the explored area

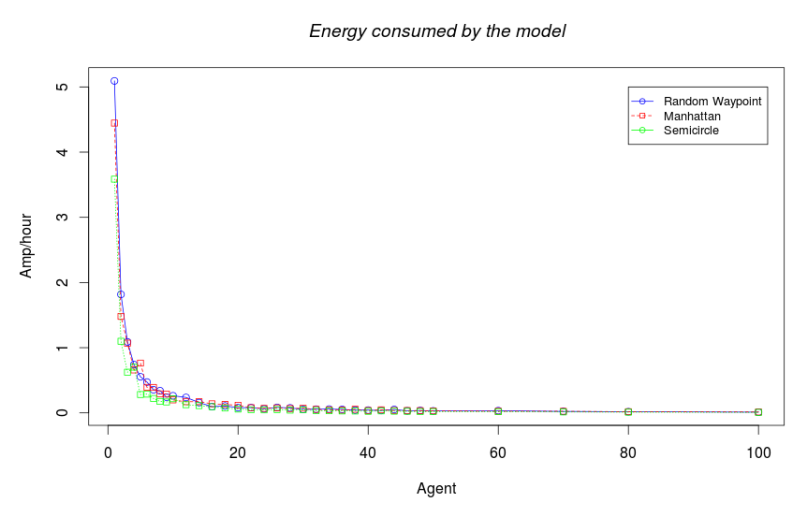

Figure 5. Energy consumed by agents with $80 \%$ convergence of the explored area

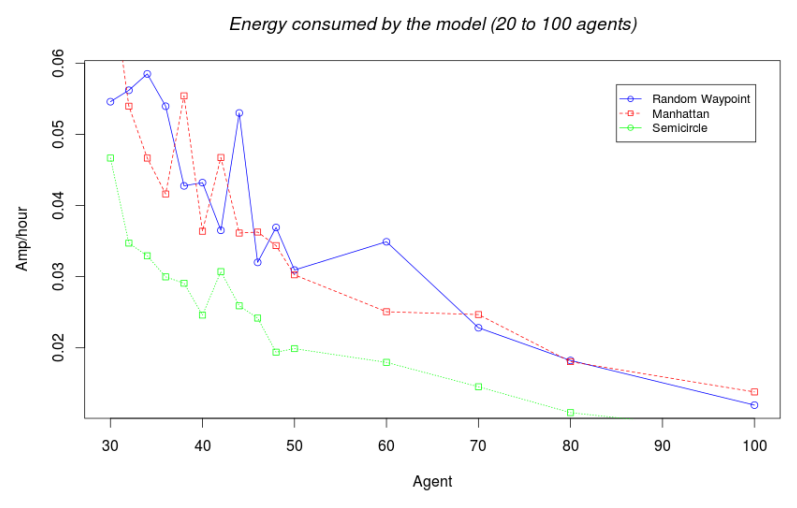

Figure 6. Magnification of Figure 5, between 20 and 100 agents

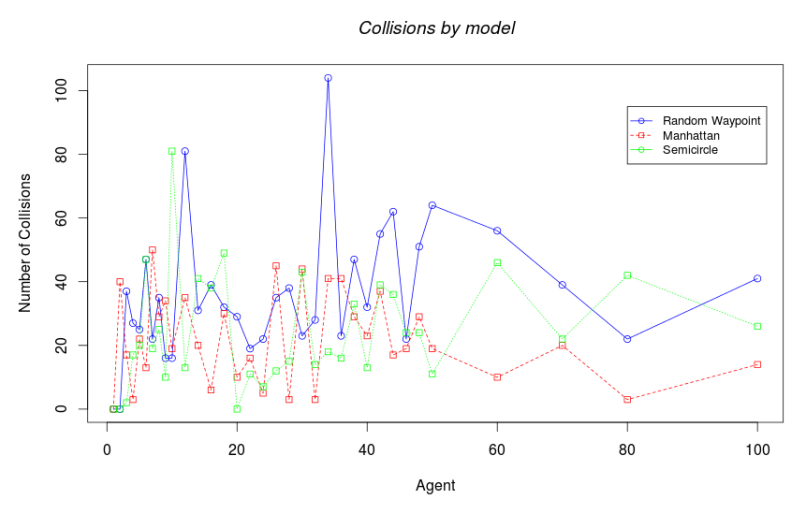

Figure 7. Number of collisions by motion model 


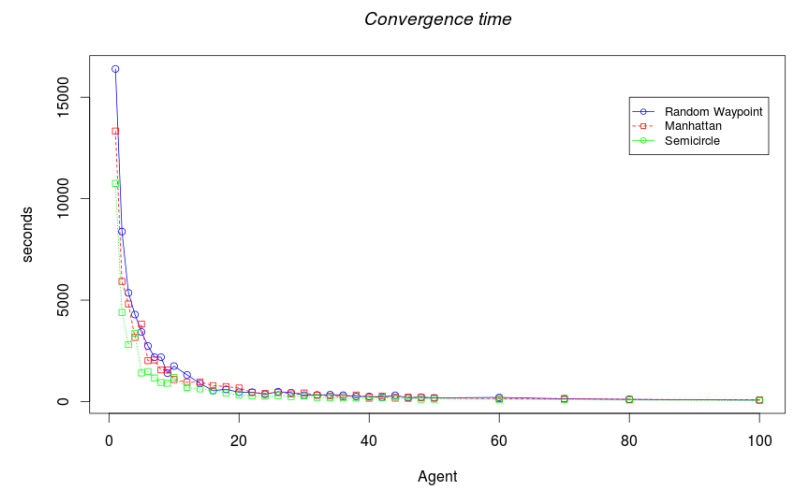

Figure 8. Convergence time by motion model

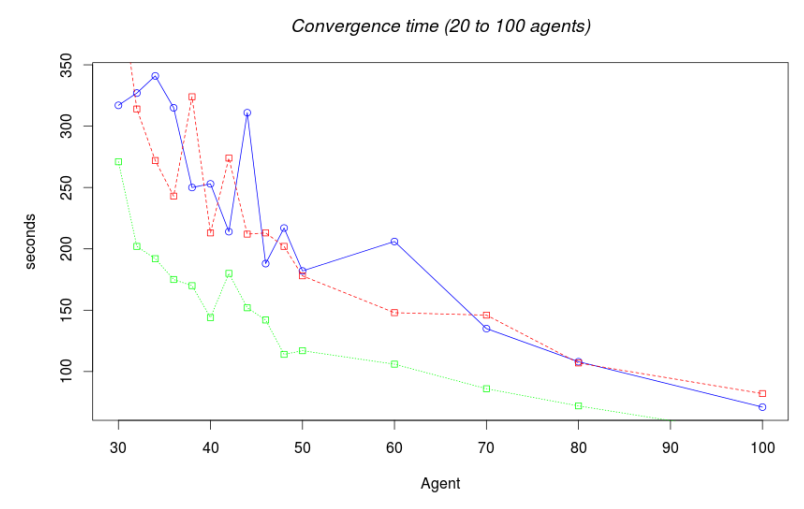

Figure 9. Magnification of Figure 8, between 20 and 100 agents

\section{CONCLUSIONS}

The analyzed data allow determining how a motion model for robots can explore an ideal flat area, unobstructed and without scanning mechanisms as the vision range of a camera or ultrasonic sensor. These are done without any cognitive intelligence and heuristics.

The semicircular motion model presents better performance than random waypoint and Manhattan models. This is in terms of accumulated distance, energy, number of collisions between robots' nodes and convergence time. It helps making this movement as a basis of future SLAM task's work for two scan types (within and outside the semicircle through perception).

The experiment allowed validating the design methodology of computational experiments founded on designbased science. It can be expanded to physical experiments for validation of computational experiments.

\section{REFERENCES}

[1] T. Kelemenová, M. Kelemen, L. Miková, V. Maxim, E. Prada, T. Lipták, and F. Menda, "Model Based Design and HIL Simulations,” Am. J. Mech. Eng., vol. 1, no. 7, pp. 276-281, Jan. 2013.

[2] Vijay K. Vaishnavi and William Kuechler, Design Science Research Methods and Patterns. CRC Press, Taylor \& Francis Group, 2007.

[3] P. Santi, "Random Waypoint Model and Wireless Network Simulation," in Mobility Models for Next Generation Wireless Networks, Chichester, UK: John Wiley \& Sons, Ltd, 2012, pp. 275291. http://dx.doi.org/10.1002/9781118344774.ch21

[4] A. Hanggoro and R. F. Sari, "Performance evaluation of the manhattan mobility model in vehicular ad-hoc networks for high mobility vehicle," in 2013 IEEE International Conference on Communication, Networks and Satellite (COMNETSAT), 2013, pp. 31-36. http://dx.doi.org/10.1109/COMNETSAT.2013.687 $\underline{0855}$

[5] Z. E. N. Y.F. Lin, "Computer Simulation Research for Infant Industry Theory based on Repast Simphony," Int. J. Online Eng., vol. 11, no. 7, 2015.

[6] J. Kerdsri and T. Veeraklaew, "Analysis of Energy and Mobility Model on Opportunistic Routing Algorithms," in Proceedings of the 11th EAI International Conference on Heterogeneous Networking for Quality, Reliability, Security and Robustness, 2015. http://dx.doi.org/10.4108/eai.19-8-2015.2260703

[7] D. Perdana, M. Nanda, R. Ode, and R. F. Sari, "Performance evaluation of PUMA routing protocol for Manhattan mobility model on vehicular ad-hoc network," in 2015 22nd International Conference on Telecommunications (ICT), 2015, pp. 80-84. http://dx.doi.org/10.1109/ict.2015.7124661

\section{AUTHORS}

John Petearson Anzola is a doctoral student of Engineering of the University Francisco José de Caldas, Faculty of Electrical Engineering of the University Foundation Los Libertadores and member of the research group GUIAS (e-mail: jpanzolaa@libertadores.edu.co).

Helien Parra Riveros., is a doctoral student of Engineering of the University Francisco José de Caldas, Faculty of Industrial Engineering of the University Foundation Los Libertadores and member of the research group GUIAS (e-mail: hparrar@libertadores.edu.co).

Andres Camilo Jimenez is a doctoral student of Engineering of the University Francisco José de Caldas, Faculty of Electrical Engineering of the University Foundation Los Libertadores and member of the research group GUIAS (e-mail: acjimeneza@libertadores.edu.co).

Submitted, 09 March 2016. Published as resubmitted by the authors on 09 April 2016. 